\title{
Characteristic nonreflecting boundary conditions for open boundaries in lattice Boltzmann methods
}

\author{
Salvador Izquierdo ${ }^{1, *}$ and Norberto Fueyo ${ }^{2, \dagger}$ \\ ${ }^{1}$ Laboratorio de Investigación en Tecnologías de la Combustión (LITEC), Centro Superior de Investigaciones Científicas (CSIC), \\ María de Luna, 10, 50018 Zaragoza, Spain \\ ${ }^{2}$ Fluid Mechanics Group and LITEC (CSIC), University of Zaragoza María de Luna 3, 50015, Zaragoza, Spain
}

(Received 18 December 2007; revised manuscript received 2 May 2008; published 24 October 2008)

\begin{abstract}
A boundary condition for lattice Boltzmann methods, based on the movement of information through Euler characteristic directions, is developed. With respect to the similar conditions used in finite-difference or finitevolume implementations, some corrections are needed to compensate the isothermal compressible nature of standard lattice Boltzmann methods for fluid flow. The results show that the proposed method for inlets and outlets is highly nonreflecting, and mass conserving.
\end{abstract}

DOI: 10.1103/PhysRevE.78.046707

PACS number(s): 47.11.-j, 47.60.-i

\section{INTRODUCTION}

The lattice Boltzmann (LB) method solves the isothermal compressible Navier-Stokes equation in the incompressible and continuous limit (low Mach and Knudsen numbers) [1]. It has been successfully used to reproduce the incompressible solution of the Navier-Stokes equations in a variety of configurations, even those with a complex coupling of physical and chemical processes [2]. It has been extensively shown that the choice of initial and boundary conditions influence the accuracy of the method $[3,4]$. However, even if the spurious behaviors of these conditions in the form of small numerical waves (wiggles) and initial layers are suppressed, it is still necessary to formulate a mechanism to treat the physical waves generated in the bulk of the fluid $[5,6]$.

When solving the fluid flow with compressible solvers, the presence of open boundaries close to vortex or pressure wave generation zones requires a mechanism for modeling the far-field environment, so that the truncation of the physical domain does not bear an influence on the behavior of the process. LB methods are compressible in nature, albeit only slightly; they have been, in fact, used to compute sound generation [7]. The correct truncation of the domain becomes a more serious problem for complex flows, e.g., turbulent combustion with acoustic coupling [8]. Additionally, this problem is related to the well-posedness of the boundary conditions. And, further, it is necessary to guarantee not only a good behavior in terms of wave suppression, but also a correct preservation of the order of the method [9].

The techniques for artificial boundary modeling may be classified in two main groups [10]: Characteristic boundary conditions and absorbing layers. We suggest that some sort of these nonreflecting boundaries should be applied for most of the simulations with LB methods, including those where it is desired to recover the incompressible solution. However, the standard LB equation is thermodynamically inconsistent [11] and some considerations must be taken into account to overcome this drawback; for example, different kinds of en-

\footnotetext{
*salvador.izquierdo@unizar.es

†norberto.fueyo@unizar.es
}

ergy conserving methods can be used $[11,12]$. In this paper we propose a formulation of a characteristic boundary condition for the standard LB method [13] which is based on the characteristics of the Euler equations and their extension to the Navier-Stokes ones; these are the so-called Navier-Stokes characteristic boundary condition (NS-CBC), developed by Poinsot and Lele [9].

\section{LATTICE BOLTZMANN METHOD}

We use a two-dimensional LB method (D2Q9) for simplicity, with a multi-relaxation-time (MRT) collision operator [14]. The extension to three dimensions and the use of other collision operators do not need additional considerations. The evolution equation for the velocity distribution functions $f$ is

$$
\boldsymbol{f}\left(x_{i}+\boldsymbol{e}_{i} \delta t, t+\delta t\right)-\boldsymbol{f}\left(x_{i}, t\right)=-\boldsymbol{M}^{-1} \cdot \boldsymbol{S} \cdot\left[\boldsymbol{m}\left(x_{i}, t\right)-\boldsymbol{m}^{\mathrm{eq}}\left(x_{i}, t\right)\right],
$$

where $\quad e_{i x}=(0,1,0,-1,0,1,-1,-1,1) \quad$ and $\quad e_{i y}$ $=(0,0,1,0,-1,1,1,-1,-1)$ are the components of the discrete set of nine microscopic velocities; $\boldsymbol{m}$ $=\left(\rho, e, \epsilon, \rho_{0} u_{x}, q_{x}, \rho_{0} u_{y}, q_{y}, p_{x x}, p_{x y}\right)^{T}$ is the macroscopic moment vector; $\boldsymbol{M}$ is the transformation matrix; $S$ $=\operatorname{diag}\left(0, s_{e}, s_{\epsilon}, 0, s_{q}, 0, s_{q}, s_{\nu}, s_{\nu}\right)$ is a diagonal matrix of relaxation factors and $\boldsymbol{m}^{\mathrm{eq}}$ are the moment equilibrium values,

$$
\begin{gathered}
e^{\mathrm{eq}}=-2(2-\kappa) \rho+\rho_{0}\left(u_{x}^{2}+u_{y}^{2}\right), \\
\epsilon^{\mathrm{eq}}=\rho+\rho_{0}\left(u_{x}^{2}+u_{y}^{2}\right), \\
q_{x}^{\mathrm{eq}}=-\rho_{0} u_{x}, \\
q_{y}^{\mathrm{eq}}=-\rho_{0} u_{y}, \\
p_{x x}^{\mathrm{eq}}=\rho_{0}\left(u_{x}^{2}-u_{y}^{2}\right),
\end{gathered}
$$

and

$$
p_{x y}^{\mathrm{eq}}=\rho_{0} u_{x} u_{y} .
$$

Equilibrium moments are considered in their incompressible formulation, whereby the density is split in a fixed reference 
value and a fluctuation, $\rho=\rho_{0}+\delta \rho$, and the $\mathbf{u} \delta \rho$ and $\mathbf{u}^{2} \delta \rho$ terms are neglected. A complete description of the MRT method applied here can be found in the paper by Lallemand and Luo [15]; and a complete overview of the LB method and its applications in Refs. [2,16].

The derivation of the Navier-Stokes system equivalent to the LB one is made through a Chapman-Enskog expansion of Eq. (1) in the low-Knudsen-number and low-Machnumber limit. Macroscopic properties are obtained by integrating the collision function over the microscopic velocity space. The speed of sound $c_{s}$ is

$$
c_{s}=\sqrt{\kappa R T}=\sqrt{\kappa \frac{p}{\rho}}
$$

where the specific-heat ratio $\kappa$ takes the value of 1 for the D2Q9 method [15], and therefore $c_{s}^{2}=1 / 3$. The transport coefficients are related to the relaxation factors, and thus the shear and bulk viscosity are, respectively,

$$
\nu=\frac{1}{3}\left(\frac{1}{s_{\nu}}-\frac{1}{2}\right)
$$

and

$$
\zeta=\frac{2-\kappa}{6}\left(\frac{1}{s_{e}}-\frac{1}{2}\right)
$$

\section{NS-CBC FOR LATTICE BOLTZMANN}

A minimal working scheme of the NS-CBC is formulated for LB based on the same procedure followed in Ref. [9]. The goal is to derive nonreflective Dirichlet boundary conditions for both the velocity and the pressure. A nonreflective Dirichlet pressure condition can be used as the far-field boundary condition usually to be applied in compressible fluid-flow simulations, but this is only an approximation to the far-field behavior. An approach to estimate real far-field conditions for incompressible fluid flows can be found in the work by Bönish et al. [17]. The local one-dimensional inviscid (LODI) equations expressed in primitive variables $\mathbf{U}$ $=\left(\rho, u_{x}, u_{y}, E\right)$ are solved at the boundary. These are the Euler equations without the transverse terms; for an open boundary normal to the $x$ direction these are

$$
\begin{gathered}
\frac{\partial \rho}{\partial t}+u_{x} \frac{\partial \rho}{\partial x}+\rho \frac{\partial u_{x}}{\partial x}=0, \\
\frac{\partial u_{x}}{\partial t}+u_{x} \frac{\partial u_{x}}{\partial x}+\frac{1}{\rho} \frac{\partial p}{\partial x}=0, \\
\frac{\partial u_{y}}{\partial t}+u_{x} \frac{\partial u_{y}}{\partial x}=0, \\
\frac{\partial \rho E}{\partial t}+\frac{\partial\left[u_{x}(\rho E+p)\right]}{\partial x}=0 .
\end{gathered}
$$

It should be noticed that the energy equation is included here for consistency, but the LB model is athermal; the conse-

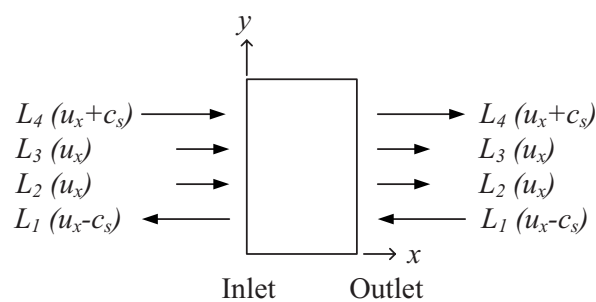

FIG. 1. Wave amplitude variations for boundaries located on the $y$ axis.

quences of this are discussed below. Expressing the LODI equations in vector form results in

$$
\frac{\partial}{\partial t} \mathbf{U}+\Gamma_{x} \frac{\partial}{\partial x} \mathbf{U}=0
$$

from which the amplitude variations $\mathbf{L}$ can be computed as $\mathbf{L}=\boldsymbol{\Lambda} \mathbf{S} \frac{\partial}{\partial x} \mathbf{U}, \mathbf{S}$ being a matrix of left eigenvectors of $\boldsymbol{\Gamma}_{x}$ and $\boldsymbol{\Lambda}$ the diagonal matrix of the eigenvalues of $\boldsymbol{\Gamma}_{x}$. Therefore, the LODI relations can be rewritten in compact form as

$$
\frac{\partial}{\partial t} \mathbf{U}+\mathbf{S}^{-1} \mathbf{L}=0 .
$$

The resulting expressions for the amplitude variations are

$$
\mathbf{L}=\left\{\begin{array}{l}
L_{1} \\
L_{2} \\
L_{3} \\
L_{4}
\end{array}\right\}=\left\{\begin{array}{c}
\left(u_{x}-c_{s}\right)\left(\frac{\partial p}{\partial x}-\rho c_{s} \frac{\partial u_{x}}{\partial x}\right) \\
u_{x} \frac{\partial u_{y}}{\partial x} \\
u_{x}\left(c_{s}^{2} \frac{\partial \rho}{\partial x}-\frac{\partial p}{\partial x}\right) \\
\left(u_{x}+c_{s}\right)\left(\frac{\partial p}{\partial x}+\rho c_{s} \frac{\partial u_{x}}{\partial x}\right)
\end{array}\right\} .
$$

Figure 1 shows the wave amplitude directions in each open boundary.

Considering the isothermal nature of the LB method used (the energy equation is suppressed), and using Eqs. (6) and (9) the LODI relations can be recast as

$$
\begin{gathered}
\frac{\partial \rho}{\partial t}+\frac{1}{2 c_{s}^{2}}\left(L_{4}+L_{1}\right)+\frac{1}{c_{s}^{2}} L_{3}=0, \\
\frac{\partial u_{x}}{\partial t}+\frac{1}{2 \rho c_{s}}\left(L_{4}-L_{1}\right)=0, \\
\frac{\partial u_{y}}{\partial t}+L_{2}=0 .
\end{gathered}
$$

The implementation of NS-CBC in the LB equation follows the same conceptual steps as for the Navier-Stokes equations: (i) Physical boundary conditions are imposed, which implies the selection of boundary conditions for the Euler equations and for the viscous terms; (ii) the wave amplitude variations L, Eqs. (9), are computed; and (iii) the conservation Eqs. (10) are solved at the boundary using the wave 


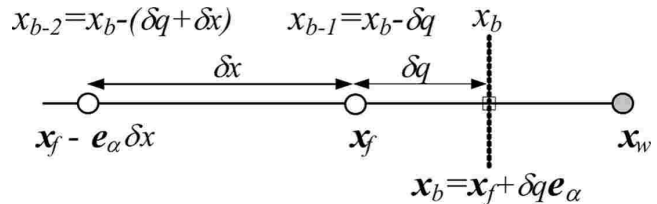

FIG. 2. The open boundary cuts the link between $\mathbf{x}_{f}$ and $\mathbf{x}_{w}$ at $\delta q$. For the Euler equations, the values on the $x$ axis are considered, $x_{b}, x_{b-1}, x_{b-2}$.

amplitudes previously computed and the viscous boundary conditions.

In the third step, the adaptation of the algorithm to the LB equation requires some discussion. As a result of the isothermal compressible nature of the LB method, an artificial bulk viscosity appears which can be modulated with the relaxation factor $s_{e}[15]$; but, additionally, the nonconservation of energy prevents the pressure wave to be completely suppressed at the boundary when the pressure is specified. To correct this effect we include the characteristic amplitude related to the energy equation in the continuity LODI equation (10a).

Considering Eq. (3), $L_{3}=0$ for $\kappa=1$ (which is the original value of $\kappa$ in the D2Q9 lattice Boltzmann method). In nature, the specific-heat ratio takes values $\kappa>1$ for the different gases depending on the degrees of freedom (e.g., $\kappa=5 / 3$ for monoatomic gases). As $\kappa$ increases, two different consequences are noteworthy: On the one hand, the bulk viscosity becomes smaller, and this cannot help suppress, via viscous dissipation, the excess of energy due to the isothermal character of the LB representation of the flow; on the other hand, the energy-related equilibrium moment $e^{\text {eq }}$ is modified, which approximates the scheme towards its lost thermodynamical consistency. Therefore, a tradeoff between these two behaviors is desirable. We have found numerically that using a value of $\kappa \approx 1.2$ in the boundary condition is the best for nonreflecting purposes. Thus, we use this specific-heat ratio (only at the boundary, and not within the fluid domain) to correct the behavior of the NS-CBC for LB.

\section{CBC IMPLEMENTATION FOR LB}

The characteristic boundary conditions $(\mathrm{CBC})$ can be applied at every boundary (inlets, outlets, walls), although the implementation details depend on the boundary type. In general, the incoming wave amplitudes need to be modeled whereas the outgoing ones are computed from the LODI relations, Eq. (10) (see Fig. 1). We describe here the implementation for open boundaries parallel to the $y$ axis, with $\delta q=1 / 2$ as this is the most common case. In Fig. 2 the nomenclature applied is shown. For link-type LB boundary conditions the work by Ginzburg et al. [18] is followed; this reference can be used also to derive higher-order extension for the boundary conditions proposed here, or to adapt them to arbitrary geometries. For the Euler equations solved at the boundary the spatial derivatives are computed in the $x$ direction instead of along the links. This avoids problems when dealing with corners. In the following, the implementation of the outflow far-field pressure condition (approximated by the nonreflecting Dirichlet-condition for pressure) and of the inflow nonreflecting Dirichlet condition for velocity are detailed.

\section{A. Outlet}

For a perpendicular outlet with $\delta q=1 / 2$ where the reference pressure is fixed $p_{b}=p\left(x_{b}\right)$, the pressure antibounceback (PAB-p) boundary condition can be applied [18],

$$
\begin{aligned}
f_{\bar{\alpha}}\left(\mathbf{x}_{f}, t+1\right)= & -\tilde{f}_{\alpha}\left(\mathbf{x}_{f}, t\right)+2 f_{\alpha}^{\mathrm{eq}+}\left(\mathbf{x}_{b}, \hat{t}\right) \\
& +\left(2-s_{\nu}\right)\left[f_{\alpha}^{+}\left(\mathbf{x}_{f}, t\right)-f_{\alpha}^{\mathrm{eq}+}\left(\mathbf{x}_{f}, t\right)\right],
\end{aligned}
$$

where $\tilde{f}_{\alpha}$ are the post-collision distribution functions, and $\bar{\alpha}$ is the opposite direction to $\alpha$. In Eq. (11) the first term is the antibounce back, the second term is for the Dirichlet pressure setting, and the last one is a correction to eliminate secondorder error terms. The symmetric equilibrium distribution functions $f_{\alpha}^{\mathrm{eq}+}$ are computed as

$$
f_{\alpha}^{\mathrm{eq}+}=\omega_{\alpha} \rho+\frac{9}{2} \omega_{\alpha} \rho_{0}\left[\left(\mathbf{e}_{\alpha} \cdot \mathbf{u}\right)^{2}-\frac{1}{3}(\mathbf{u} \cdot \mathbf{u})\right] .
$$

When $f_{\alpha}^{\text {eq+ }}$ are used for the error correction term at $\left(\mathbf{x}_{f}, t\right)$, values are stored during the collision step to be used during boundary-condition implementation at post-collision step. The same stands for the symmetric distribution functions

$$
f_{\alpha}^{+}=\frac{1}{2}\left(f_{\alpha}+f_{\bar{\alpha}}\right)
$$

To impose the Dirichlet pressure condition, $f_{\alpha}^{\mathrm{eq}+}$ at $\left(\mathbf{x}_{b}, \hat{t}\right)$ must be computed using the desired $\rho_{b}=p_{b} c_{s}^{-2}$ and the actual velocity values, which are approximated as

$$
\mathbf{u}\left(\mathbf{x}_{b}, \hat{t}\right) \approx \mathbf{u}\left(\mathbf{x}_{b-1}, t\right)+\frac{1}{2}\left[\mathbf{u}\left(\mathbf{x}_{b-1}, t\right)-\mathbf{u}\left(\mathbf{x}_{b-2}, t\right)\right] .
$$

The time $\hat{t}$ is a notional intermediate time for the conditions to be set at time $t$. For PAB $\hat{t}=t+1 / 2$. It must be noticed that Eq. (11) is strictly valid for the two-relaxation time model $\left(s_{\nu}=s_{e}=s_{\epsilon}\right)$ and for incompressible fluid flows. For the MRT collision operator, the correction term is computed by projecting $\left(f_{\alpha}^{+}-f_{\alpha}^{\mathrm{eq}+}\right)$ on the symmetric eigenvectors and then multipliying each momentum by its respective eigenvalue.

To introduce the $\mathrm{CBC}$ in the $\mathrm{PAB}$ scheme, alternative values must be computed at $\left(\mathbf{x}_{b}, \hat{t}\right)$ for $\rho$ and $\mathbf{u}$ according to the LODI relations. The values for density (pressure) and velocity computed using LODI relations are enclosed in angular brackets $\left(\langle\rho\rangle\right.$ and $\left.\left\langle u_{i}\right\rangle\right)$, and the boundary condition is denoted as PAB-CBC. We proceed first by computing the outgoing wave amplitudes $\left(L_{2}, L_{3}\right.$, and $\left.L_{4}\right)$ from Eq. (9). A second-order backward stencil is used for the unknown spatial derivatives,

$$
\begin{aligned}
\frac{\delta \phi}{\delta x}\left(x_{b}, \hat{t}-1\right) \approx & \frac{1}{3}\left[8 \phi\left(x_{b}, \hat{t}-1\right)-9 \phi\left(x_{b-1}, \hat{t}-1\right)\right. \\
& \left.+\phi\left(x_{b-2}, \hat{t}-1\right)\right] .
\end{aligned}
$$

The incoming amplitude $L_{1}$ is approached by the means of a linear relaxation model

$$
L_{1}\left(\mathbf{x}_{b}, \hat{t}-1\right)=k_{1}\left[\left\langle p\left(\mathbf{x}_{b}, \hat{t}-1\right)\right\rangle-p_{b}\right],
$$

where 


$$
k_{1}=\sigma_{1}\left(1-\mathrm{Ma}^{2}\right) \frac{c_{s}}{L}
$$

being $L$ the length between inlet and outlet. A value of $\sigma_{1}$ $=0$ means that no information is coming into the domain; however, this does not allow us to properly impose the reference pressure. To eliminate the pressure reflection and, at the same time, fix the reference pressure, $\sigma_{1}$ should be in the range $0.58<\sigma_{1}<\pi$ [19]. Consequently, the boundary condition becomes partially reflective.

To explicitly solve the LODI relations at $\mathbf{x}_{b}$, Eqs. (10), a first-order time-derivative stencil is applied,

$$
\begin{gathered}
\langle\rho(\hat{t})\rangle \approx\langle\rho(\hat{t}-1)\rangle-\frac{\delta t}{2 c_{s}^{2}}\left[L_{4}(\hat{t}-1)+L_{1}(\hat{t}-1)\right]-\frac{1}{c_{s}^{2}} L_{3}(\hat{t}-1), \\
\left\langle u_{x}(\hat{t})\right\rangle \approx\left\langle u_{x}(\hat{t}-1)\right\rangle-\frac{\delta t}{2 \rho c_{s}}\left[L_{4}(\hat{t}-1)-L_{1}(\hat{t}-1)\right], \\
\left\langle u_{y}(\hat{t})\right\rangle \approx\left\langle u_{y}(\hat{t}-1)\right\rangle-\delta t L_{2}(\hat{t}-1) .
\end{gathered}
$$

\section{B. Inlet}

For an inlet where a velocity $\mathbf{u}_{b}$ is imposed, the velocity bounce-back boundary (UBB- $\left.\rho_{0} u_{0}\right)$ condition can be applied [20],

$$
f_{\bar{\alpha}}\left(\mathbf{x}_{f}, t+1\right)=\tilde{f}_{\alpha}\left(\mathbf{x}_{f}, t\right)-2 f_{\alpha}^{\mathrm{eq}-}\left(\mathbf{x}_{b}, \hat{t}\right) .
$$

For this condition no error-correction term is needed. The antisymmetric equilibrium function is computed as

$$
f_{\alpha}^{\mathrm{eq}-}=3 \omega_{\alpha} \rho_{0}\left(\mathbf{e}_{\alpha} \cdot \mathbf{u}\right),
$$

where $\mathbf{u}$ is the prescribed value at $\left(\mathbf{x}_{b}, \hat{t}\right)$, with $\hat{t}=t+1 / 2$.

To incorporate the CBC into the UBB scheme (UBB$\mathrm{CBC}$ ) the outgoing wave amplitude $L_{1}$ is computed from Eq. (9) using second-order forward spatial derivatives,

$$
\begin{aligned}
\frac{\delta \phi}{\delta x}\left(x_{b}, \hat{t}-1\right) \approx & \frac{1}{3}\left[-8 \phi\left(x_{b}, \hat{t}-1\right)+9 \phi\left(x_{b-1}, \hat{t}-1\right)\right. \\
& \left.-\phi\left(x_{b-2}, \hat{t}-1\right)\right] .
\end{aligned}
$$

The incoming wave amplitudes $\left(L_{2}, L_{3}\right.$, and $\left.L_{4}\right)$ are approached using linear relaxation models as for the outlet,

$$
L_{i}\left(\mathbf{x}_{b}, \hat{t}-1\right)=k_{i}\left[\left\langle u\left(\mathbf{x}_{b}, \hat{t}-1\right)\right\rangle-u_{b}\right], \quad i=2,3,4,
$$

where $k_{i}$ can be computed as in Eq. (17), and the ensuing discussion about $\sigma_{i}$ also holds.

\section{TEST CASES FOR CBC}

Three cases are presented to test the different desirable properties of open boundary conditions. A one-dimensional pressure wave is simulated to check the difference between usual reflective boundary conditions $\left(\mathrm{UBB}-\rho_{0} u_{0}\right.$ for inlets and PAB- $p$ for outlets) and nonreflective ones (UBB-CBC and $\mathrm{PAB}-\mathrm{CBC}$ with $k_{i}=0$ ); a laminar two-dimensional channel is used to quantify the mass balance within the domain due to the application of different boundary conditions; and the unsteady laminar two-dimensional flow around a square cylinder is used to evaluate the benefit of using $\mathrm{CBC}$ in realistic geometries. For simplicity, in the following we use UBB-PAB to refer to the boundary condition using $\mathrm{UBB}-\rho_{0} u_{0}$ for inlets and PAB- $p$ for outlets, and CBC to refer to the boundary condition using UBB-CBC for inlets and PAB-CBC for outlets.

\section{A. One-dimensional wave}

A two-dimensional one-directional flow in which a pressure perturbation is introduced at $t=0$ is simulated. The boundary conditions used are at the inlet, a uniform velocity $u_{x}=u_{0}$ is imposed; at the outlet, a reference density, $p_{0}$ $=c_{s}^{2} \rho_{0}=1 / 3$ is fixed; and the upper and lower boundaries have periodic boundary conditions. The flow is characterized by a Mach number $\mathrm{Ma}=0.1$ and a Reynolds number $\mathrm{Re}$ $=1.732$ based on the lattice space unit. The domain length in lattice units is $N_{x}=2500\left(N_{y}=10\right)$. As initial conditions, a uniform velocity field of $u_{x}=u_{0}$ and a Gaussian pressure pulse in the middle of the domain are imposed,

$$
p=p_{0}+\left(p_{\max }-p_{0}\right) \exp \left(\frac{-\left(X-X_{0}\right)^{2}}{2 s^{2}}\right),
$$

with the values selected for the free parameters being $p_{\max }$ $=\rho_{\max } c_{s}^{2}=1.1 c_{s}^{2}, X_{0}=0.5$, and $s=50$. The velocity distribution functions are initially computed from their equilibrium distribution functions.

The variables used to report the results are a dimensionless time $T=t\left(c_{s} / N_{x}\right)$, and a dimensionless coordinate $X$ $=x / N_{x}$. All the simulations presented use the following relaxation factors: $s_{\nu}$ takes the value dictated by the resolution and the Reynolds and Mach numbers; $s_{e}=s_{\epsilon}=s_{\nu}$ unless otherwise specified below; and $s_{q}=8\left(2-s_{\nu}\right) /\left(8-s_{\nu}\right)$ according to the relation stated in [18] (with $\Lambda_{e o}=3 / 16$ ) to reduce the error at the boundaries. For inlets and outlets we apply equilibrium boundary conditions to recover first-order accuracy, and UBB at inlets and PAB at outlets to obtain second-order boundary conditions. For $\mathrm{CBC}$ we use $k_{i}=0$ and $\kappa=1$ unless some other value is specified.

To show the behavior of the proposed boundary condition and to assess the gain obtained, we plot in Fig. 3 the temporal evolution of the initial pressure peak for different inletoutlet boundary conditions. For all cases the peak splits as expected into two different pressure waves which travel at $u_{0}+c_{s}$ and $u_{0}-c_{s}$, respectively.

For the equilibrium boundary condition, these pressure waves are completely reflected at the boundaries, changing their phase when they reach the fixed-pressure boundary condition. When adding the viscous correction at boundaries (UBB and PAB boundary conditions), the degree of reflection may become even greater. The use of $\mathrm{CBC}$ partially suppresses the reflection at inlet and outlet; and when the $\kappa$-modified CBC is applied, the performance of the scheme is further improved. 


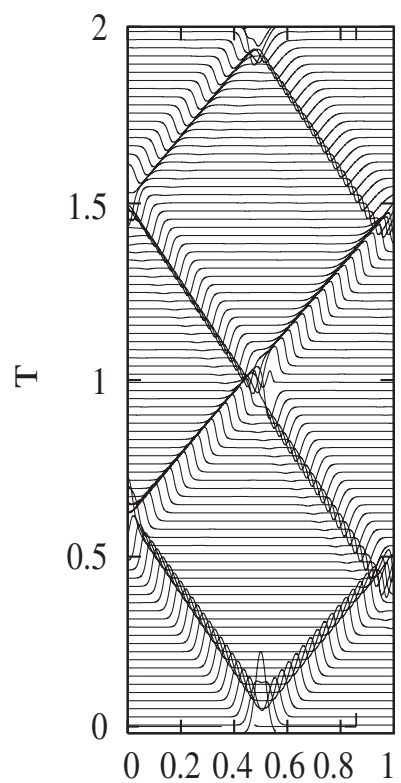

(a)

X

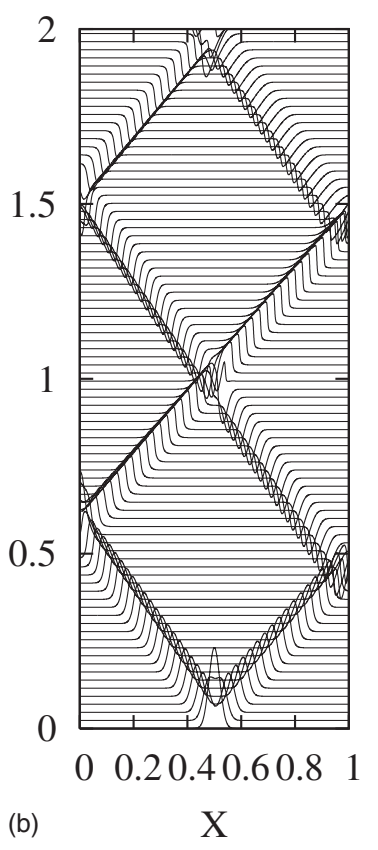

(b)

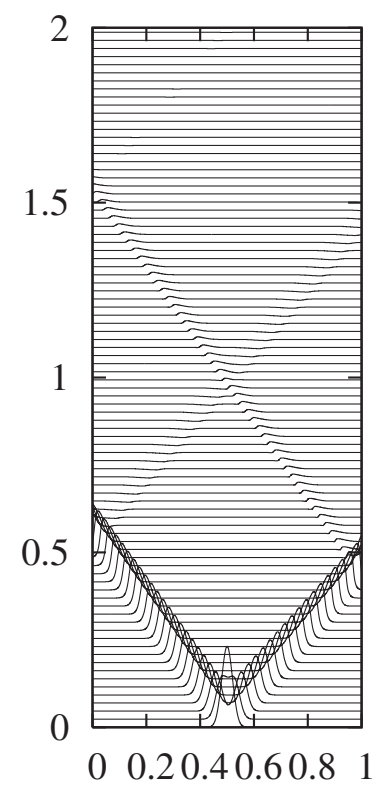

(c)

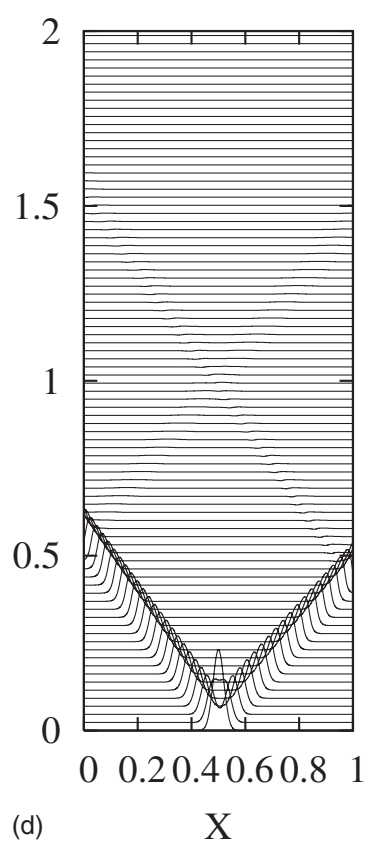

FIG. 3. Spatial-temporal evolution of the pressure. The inlet and outlet boundary conditions applied are (a) first order; (b) inlet, UBB; outlet, PAB; (c) CBC with $\kappa=1.0$ and $s_{e}=s_{\nu}$; and (d) CBC with $\kappa=1.2$ and $s_{e}=0.65$.

To quantify the performance of the boundary conditions, the degree of reflection $r$ is defined as

$$
|r|=\frac{\left|L^{+}\right|}{\left|L^{-}\right|},
$$

where $L^{+}$is the incoming wave amplitude and $L^{-}$is the outgoing one.

The influence of $s_{e}$ through the bulk viscosity Eq. (5) can be used to reduce the reflection ratio. The smallest reflection is found numerically to occur when $s_{e} \approx 1 / s_{\nu}$, Fig. 4. Results show that $|r|$ is almost independent of the reference velocity and of the maximum value of the pressure peak; and the influence of $s_{e}$ is noticed mainly at inlets.
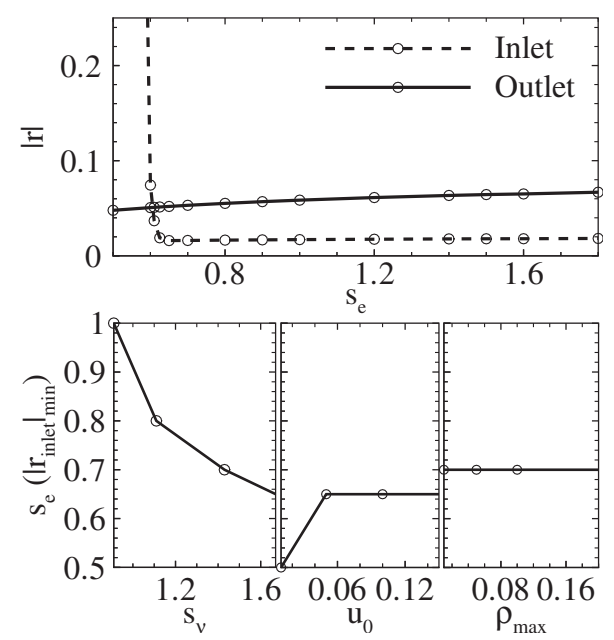

FIG. 4. Reflection ratio as a function of $s_{e}$ (top); and optimal $s_{e}$ values (bottom) depending on the viscous relaxation factor, the reference velocity and the initial amplitude of the pressure pulse.
The best-performing value of $\kappa$ has been selected by carrying out several numerical tests (see Fig. 5), resulting in an optimal value in the range $1.0<\kappa<1.3$, which turns out to be slightly dependent on the viscous relaxation factor, on the reference velocity, and also on the amplitude of the pressure wave arriving at the boundary. There is no influence of $\kappa$ at the inlet as it does not appear in the definition of the boundary.

From the test case presented, we can conclude that the use of $\mathrm{CBC}$ in athermal lattice Boltzmann methods can reduce the reflection up to $|r|=5 \%$; and this reflection can be further reduced up to $|r|=1 \%$ using the $\kappa$-modified CBC.
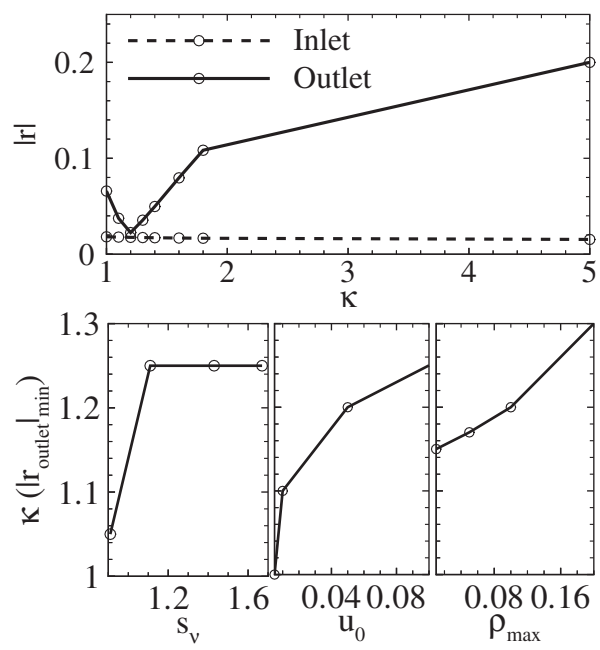

FIG. 5. Reflection ratio as a function of $\kappa$ (top); and optimal $\kappa$ values (bottom) for different values of the viscous relaxation factor, the reference velocity and the initial value of the pressure pulse. 


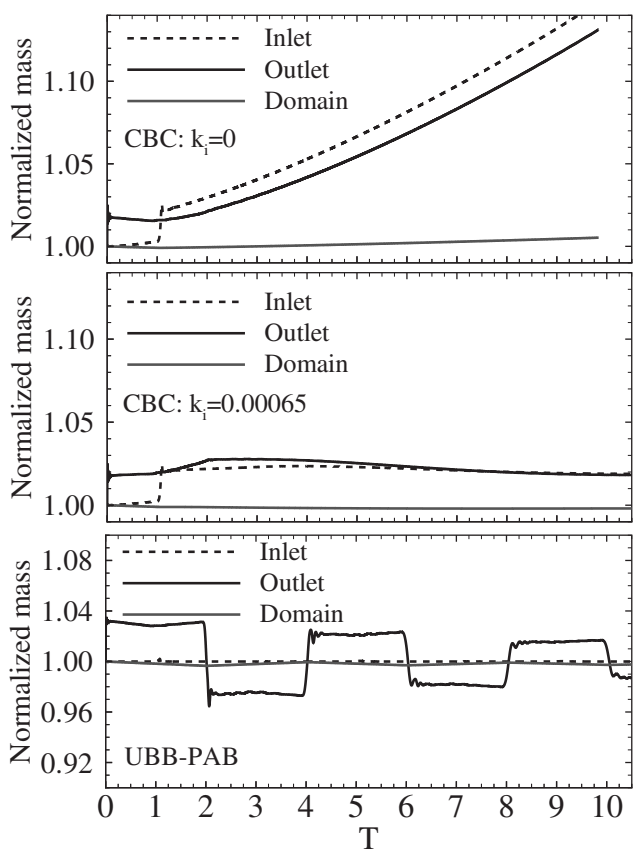

FIG. 6. Temporal evolution of the normalized mass through the inlet and the outlet and within the domain for (top) CBC with $k_{i}$ $=0$; (middle) $\mathrm{CBC}$ with $k_{i}=0.00065$; and (bottom) UBB-PAB boundary conditions

\section{B. Laminar channel}

A two-dimensional laminar channel with a parabolic velocity profile at the inlet and a reference pressure at the outlet is simulated to evaluate the mass imbalance in the fluid domain when the CBC is applied. The channel dimensions are $N_{x}=520 \delta x$ and $N_{y}=160 \delta x$, and is simulated at $\mathrm{Re}=800$ and $\mathrm{Ma}=0.087$. The domain is initializated with $u=0.0, \rho_{0}=1.0$ and using equilibrium distribution functions. To evaluate the mass imbalance, the temporal evolution of three quantities is reported in Fig. 6: The total mass leaving the domain at the outlet as the sum of the outgoing distribution functions; the total mass coming into the domain at the inlet; and the total amount of mass in the entire fluid domain. The incoming and outgoing mass fluxes are normalized with the incoming mass flow through the inlet at $T=0$; and the total mass in the domain is normalized with its value at $T=0$. The temporal coordinate is defined as $T=t\left(c_{s} / N_{x}\right)$. The optimal values for $\kappa$ and $s_{e}$ found above are used when the CBC is applied.

Three different boundary-condition sets have been compared in Fig. 6: (i) Nonreflecting $\mathrm{CBC}$ with $k_{i}=0$, which is not able to properly fix the reference values and, consequently, the mass in the domain progressively increases without bounds until the simulation fails; (ii) the partially reflecting $\mathrm{CBC}$ with the value for $k_{i}$ derived from the lower limit of Eq. (17), which is the only set of boundary conditions which is able to suppress pressure reflections and preserve mass, providing a faster convergence; and (iii) the Dirichlet velocity and pressure boundary conditions UBB and PAB, respectively, which generate an oscillation around the reference value of the total mass within the domain due to pressure wave reflection.

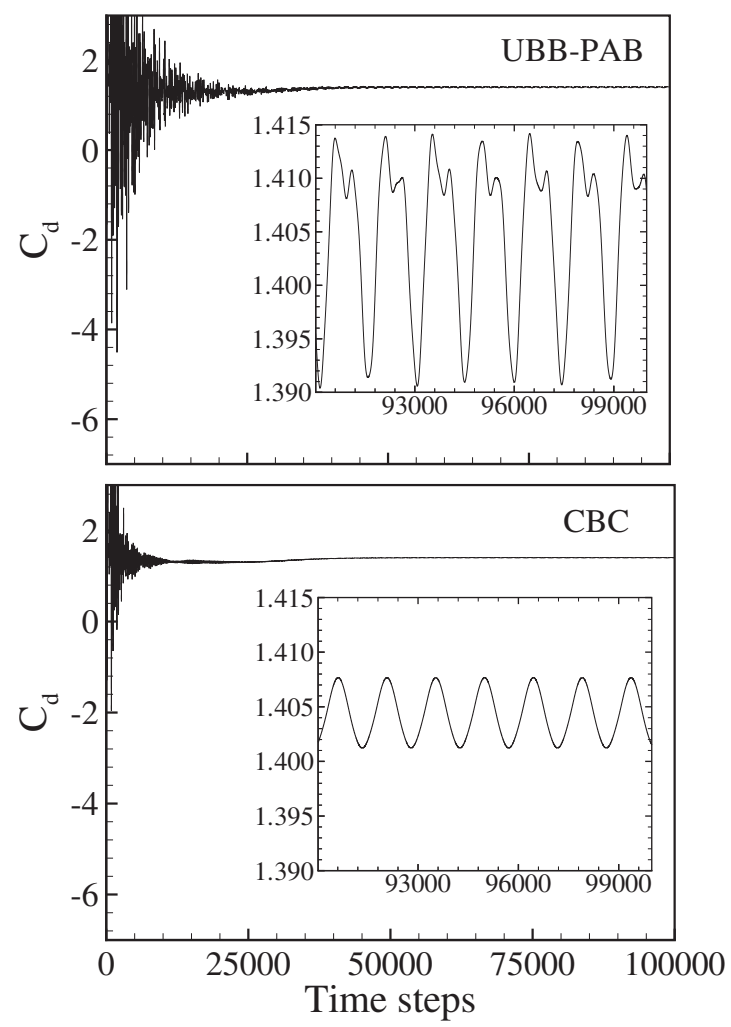

FIG. 7. Temporal evolution of the drag coefficient of the square cylinder for two different sets of open boundaries: UBB-PAB (above) and optimal CBC (below). The periodic steady state is magnified in the inset.

\section{Vortex shedding}

The two-dimensional unsteady flow around a confined square cylinder at $\mathrm{Re}=100$ and $\mathrm{Ma}=0.087$ is simulated to evaluate the behavior of the $\mathrm{CBC}$ versus the UBB-PAB in more complex configurations. The domain dimensions are $N_{x}=26 N_{s}$ and $N_{y}=8 N_{s}$, with $N_{s}=20$ being the number of nodes which discretize the side of the square. The square is deliberately placed far from the inlet to avoid any interaction, and close to the outlet to analyze the effect of the interaction between the vortices generated in the wake of the cylinder and the outlet. The distance between the downstream face of the square and the outlet is $13.5 N_{s}$, which is much less than $40 N_{s}$ as usually applied to avoid the interaction.

Two remarks can be made from the comparison between $\mathrm{CBC}$ and UBB-PAB in this test case. The first and main point is that the interaction of the vortices with the outlet results in the reflection to pressure waves which deteriorate the solution: See, for example, the shape of the time evolution of $C_{d}$ at the periodic steady state in Fig. 7. The pressurewave reflection between inlet and outlet affects the pressure pattern in the flow. It can be observed in Fig. 8 that when a vortex is shed from the cylinder the pressure is higher behind the cylinder when UBB-PAB is applied; additionally, unphysical pressure patterns appear, generating high-pressure and low-pressure zones near walls. The undesired influence of this interaction can be supported also by the peak which appears in the Fourier transform of the $C_{d}$ signal at frequen- 


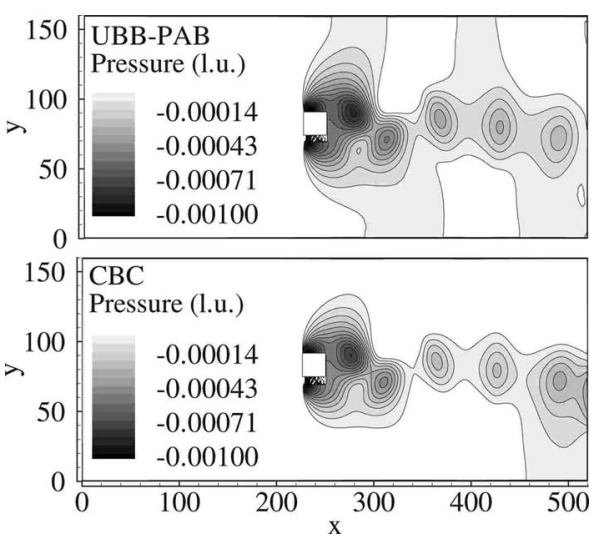

FIG. 8. Pressure isocontours (in lattice units, l.u.) at the same time step $(t=95200 \delta t)$ for two sets of open boundaries: UBB-PAB (above) and optimal CBC (below).

cies different from the one related to the vortex shedding, Fig. 9.

The second remark concerns the greater amplitude of $C_{d}$ when UBB-PAB conditions are used, Fig. 7. This is caused by (numerical) resonance appearing as the vortex shedding frequency corresponds to a natural mode of the domain [6]. The increased amplitude appears as a higher peak for the vortex shedding frequency in Fig. 9.

\section{CONCLUSIONS}

A second-order (for $\delta q=1 / 2$ ) highly nonreflective boundary condition for athermal LB methods, which does not need additional absorbing layers nor extended domains, is presented; and a detailed description of the implementation is provided for Dirichlet velocity and pressure conditions. The proposed boundary condition is expected to be useful not only for the direct simulation of the acoustic field, but also

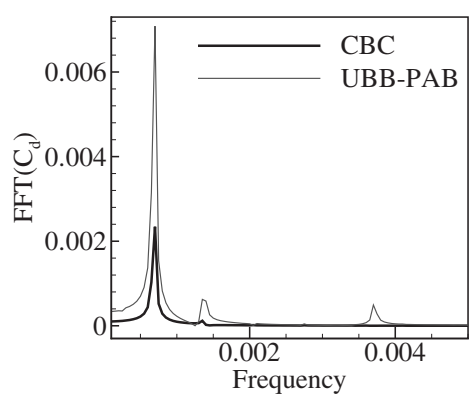

FIG. 9. Fast Fourier transform of the $C_{d}$ signal for the periodic flow using both sets of boundary conditions of Fig. 7.

for many laminar and turbulent simulations in which pressure waves interact with boundaries.

For the development of the new characteristic boundary conditions we suggest that the best choice of primitive variables is $\mathbf{U}=\left(\rho, u_{x}, u_{y}\right)$. The approach presented here can be improved in several ways from the point of view of the Euler characteristics (e.g., [21]). Possible improvements of the implementation in lattice Boltzmann methods include the following: The projection of the Euler characteristic treatment onto the equilibrium moments to express this boundary condition in the LB variables $f$ (e.g., as a correction term for $\mathrm{UBB}$ or $\mathrm{PAB}$ ); the extension, following [18], of the CBC to higher order and to arbitrary geometries (i.e., arbitrary $\delta q$ values); and the application of advanced LB methods [11] to introduce the energy-acoustic effects in a consistent way.

\section{ACKNOWLEDGMENTS}

We are very grateful to Dr. Benedicte Cuenot of CERFACS, Toulouse, France, for crucially enlightening discussions on the NS-CBC method. S.I. is supported by the I3P-CSIC Programme financed by the European Social Fund. This work was partially funded by the Spanish Government under Contract No. ENE2007-67217/ALT.
[1] S. Chen and G. Doolen, Annu. Rev. Fluid Mech. 30, 329 (1998).

[2] S. Succi, The Lattice Boltzmann Equation for Fluid Dynamics and Beyond (Oxford University Press, New York, 2001).

[3] R. Mei, L. S. Luo, P. Lallemand, and D. d'Humière, Comput. Fluids 35, 855 (2006).

[4] M. Junk and Z. Yang, J. Stat. Phys. 121, 3 (2005).

[5] P. Martínez-Lera, S. Izquierdo, and N. Fueyo, in Complex Effects in Large Eddy Simulations, Lect. Notes Comput. Sci. Eng. Vol. 56 (Springer, New York, 2007), pp. 203-217.

[6] S. Izquierdo, P. Martínez-Lera, and N. Fueyo, Analysis of Open Boundary Effects in Unsteady Lattice Boltzmann Simulations, Computers and Mathematics with Applications (to be published).

[7] A. Wilde, Comput. Fluids 35, 986 (2006).

[8] T. Poinsot and D. Veynante, Theoretical and Numerical Combustion (Edwards, Philadelphia, 2001).

[9] T. Poinsot and S. K. Lele, J. Comput. Phys. 101, 104 (1992).
[10] T. Colonius, Annu. Rev. Fluid Mech. 36, 315 (2004).

[11] S. Ansumali and I. V. Karlin, Phys. Rev. Lett. 95, 260605 (2005).

[12] E. W. S. Kam, R. M. C. So, and R. C. K. Leung, AIAA J. 45, 1703 (2007).

[13] Y. Qian, D. d'Humières, and P. Lallemand, Europhys. Lett. 17, 479 (1992).

[14] D. d'Humières, Prog. Astronaut. Aeronaut. 59, 450 (1992).

[15] P. Lallemand and L. S. Luo, Phys. Rev. E 61, 6546 (2000).

[16] D. Yu, R. Mei, L. S. Luo, and W. Shyy, Prog. Aerosp. Sci. 39, 329 (2003).

[17] S. Bönisch, V. Heuveline, and P. Wittwer, J. Math. Fluid Mech. 7, 85 (2005).

[18] I. Ginzburg, F. Verhaeghe, and D. d'Humières, Comm. Comp. Phys. 3, 427 (2008).

[19] L. Selle, F. Nicoud, and T. Poinsot, AIAA J. 42, 958 (2004).

[20] A. J. C. Ladd, J. Fluid Mech. 271, 285 (1994).

[21] R. Prosser, J. Comput. Phys. 207, 736 (2005). 